\title{
MATRIX MOMENT PERTURBATIONS AND THE INVERSE SZEGÖ MATRIX TRANSFORMATION
}

\author{
EDINSON FUENTES AND LUIS E. GARZA
}

\begin{abstract}
Given a perturbation of a matrix measure supported on the unit circle, we analyze the perturbation obtained on the corresponding matrix measure on the real line, when both measures are related through the Szegö matrix transformation. Moreover, using the connection formulas for the corresponding sequences of matrix orthogonal polynomials, we deduce several properties such as relations between the corresponding norms. We illustrate the obtained results with an example.
\end{abstract}

\section{INTRODUCTION}

The term moment problem was used for the first time in T. J. Stieltjes' classic memoir 32 (published posthumously between 1894 and 1895) dedicated to the study of continued fractions. The moment problem is a question in classical analysis that has produced a rich theory in applied and pure mathematics. This problem is beautifully connected to the theory of orthogonal polynomials, spectral representation of operators, matrix factorization problems, probability, statistics, prediction of stochastic processes, polynomial optimization, inverse problems in financial mathematics and function theory, among many other areas. In the matrix case, M. Krein was the first to consider this problem in [21, and later on some density questions related to the matrix moment problem were addressed in [14, 15, 24, 25]. Recently, the theory of the matrix moment problem is used in 10] for the analysis of random matrix-valued measures. Since the matrix moment problem is closely related to the theory of matrix orthogonal polynomials, M. Krein was the first to consider these polynomials in [22]. Later, several researchers have made contributions to this theory until today. In the last 30 years, several known properties of orthogonal polynomials in the scalar case have been extended to the matrix case, such as algebraic aspects related to their zeros, recurrence relations, Favard type theorems, and Christoffel-Darboux formulas, among many others.

2010 Mathematics Subject Classification. 42C05, 33C47.

Key words and phrases. Moment problem; Matrix moment perturbation; Matrix orthogonal polynomials; Inverse Szegö matrix transformation.

The work of the second author was partially supported by Dirección General de Investigación Científica y Técnica, Ministerio de Economía y Competitividad of Spain, under grant MTM201565888-C4-2-P and by México's Consejo Nacional de Ciencia y Tecnología (Conacyt) grant 287523. 
A nice summary, as well as many references, can be found in [8. As in the scalar case, matrix orthogonal polynomials have proved to be a useful tool in the analysis of many problems of mathematics such as differential equations [13, 28, rational approximation theory [20, spectral theory of Jacobi matrices [1, 29], analysis of polynomial sequences satisfying higher order recurrence relations [11, 17, quantum random walks [3, and Gaussian quadrature formulas [2, 12, 16, 31, among many others. In this contribution, we are interested in the study of some properties related with a perturbation of a sequence of matrix moments, within the framework of the theory of matrix orthogonal polynomials both on the real line and on the unit circle.

The structure of the manuscript is as follows. In Section 2, we state some properties of matrix orthogonal polynomials on the real line and on the unit circle that will be used in the sequel. Section 3 deals with the so-called Szegö matrix transformation and some of its properties. This has been previously studied in [8, 9, 33]. Then, we introduce a perturbation on a matrix measure supported on the unit circle that results in a perturbation of the corresponding sequence of matrix moments, and analyze the resulting perturbation on the associated matrix measure on the real line, when both measures are related through the Szegö transformation. This is the main contribution of our manuscript. Sections 4 and 5 deal with connection formulas and some other properties associated with the corresponding matrix orthogonal polynomials sequences on the real line and on the unit circle, respectively. Connection formulas for perturbations of matrix moments have been studied previously in [7, 6]. Finally, we illustrate the obtained results with an example in Section 6.

\section{MATRIX ORTHOGONAL POLYNOMIALS}

We will use the following notation. Let $\mathcal{M}_{l}=\mathcal{M}_{l}(\mathbb{C})$ be the ring of $l \times l$ matrices with complex entries. The matrices $\mathbf{I}$ and $\mathbf{0}$ will denote the $l \times l$ identity matrix and zero matrix, respectively. An Hermitian matrix $A \in \mathcal{M}_{l}$ satisfies $A=A^{H}$ (its conjugate transpose), and the set of Hermitian matrices is a real vector space. We say $A$ is positive definite if $u^{H} A u>0$ for every nonzero vector $u \in \mathbb{C}^{l}$, and positive semi-definite if the inequality is not strict. For $0 \leq k \leq j-1$, let

$$
\chi_{k}^{(j)}:=\left[\begin{array}{lllll}
\mathbf{0} & \cdots & \mathbf{I} & \mathbf{0} & \cdots
\end{array}\right]^{H} \in \mathcal{M}_{j l \times l},
$$

where $\mathbf{I}$ is in the $k$-th position, and define $\chi_{k}^{(j)}:=[\mathbf{0}, \ldots, \mathbf{0}]^{H}$ for $j \geq k$ or $k<0$. We also define

$$
\Lambda_{n, m}^{(j+1)}:=\left[\chi_{n}^{(j+1)} \chi_{n+1}^{(j+1)} \cdots \chi_{m}^{(j+1)}\right] \in \mathcal{M}_{(j+1) l \times(m-n+1) l},
$$

with $n \leq m$, for $n, m \in \mathbb{Z}$.

An $l \times l$ matrix-valued measure (or simply, matrix measure) $\sigma=\left(\sigma_{i, j}\right)_{i, j=1}^{l}$ supported on $E \subseteq \mathbb{R}$ is said to be Hermitian (resp. positive semi-definite, positive definite), if for every Borel subset $\mathcal{B}$ on $E$, the matrix $\sigma(\mathcal{B})$ is a Hermitian (resp. positive semi-definite, positive definite) matrix. Notice that the assumption of positive definiteness implies the Hermitian character of the measure. For more information about matrix measures, see [8] 
2.1. Matrix orthogonal polynomials on the real line. Let $\mu=\left(\mu_{i, j}\right)_{i, j=1}^{l}$ be a Hermitian matrix measure supported on $E \subseteq \mathbb{R}$ and denote by $\mathbb{P}^{l}[x]$ the linear space of polynomials in $x \in \mathbb{R}$ with coefficients in $\mathcal{M}_{l}$. If the scalar measures $\mu_{i, j}, i, j=$ $1, \ldots, l$, are absolutely continuous with respect to the scalar Lebesgue measure $d x$, then according to the Radon-Nikodym theorem, it can always be expressed as $d \mu_{i, j}(x)=\omega_{i, j}(x) d x$. Since $\mu$ is Hermitian, the matrix $\omega(x)=\left(\omega_{i, j}(x)\right)_{i, j=1}^{l}$ is also a Hermitian matrix, and thus

$$
d \mu(x)=\omega(x) d x .
$$

Notice that $\mu$ induces right and left sesquilinear forms, defined respectively by

$$
\begin{aligned}
\langle f, g\rangle_{R, \mu} & =\int_{E} f(x)^{H} d \mu(x) g(x) \in \mathcal{M}_{l}, \quad f, g \in \mathbb{P}^{l}[x], \\
\langle f, g\rangle_{L, \mu} & =\int_{E} f(x) d \mu(x) g(x)^{H} \in \mathcal{M}_{l}, \quad f, g \in \mathbb{P}^{l}[x] .
\end{aligned}
$$

On the other hand, the $j$-th moment associated to the matrix measure $\mu$ is defined by

$$
\mu_{j}=\left\langle\mathbf{I}, x^{j} \mathbf{I}\right\rangle_{R, \mu}=\left\langle x^{j} \mathbf{I}, \mathbf{I}\right\rangle_{L, \mu}=\int_{E} x^{j} d \mu(x) \in \mathcal{M}_{l}, \quad j \geq 0 .
$$

Notice that every moment is a Hermitian matrix, i.e., $\mu_{j}^{H}=\mu_{j}$ for $j \geq 0$, since $\mu$ is Hermitian. We denote by $H_{j} \in \mathcal{M}_{l j}$ and $F_{j} \in \mathcal{M}_{l(j+1)}$ the block matrices

$$
H_{j}=\left[\begin{array}{cccc}
\mu_{0} & \mu_{1} & \cdots & \mu_{j-1} \\
\mu_{1} & \mu_{2} & \cdots & \mu_{j} \\
\vdots & \vdots & \vdots & \vdots \\
\mu_{j-1} & \mu_{j} & \cdots & \mu_{2 j-2}
\end{array}\right], \quad F_{j}=\left[\begin{array}{cccc} 
& & & \mu_{j} \\
& H_{j} & & \vdots \\
& & & \mu_{2 j-1} \\
\mathbf{I} & x \mathbf{I} & \cdots & x^{j} \mathbf{I}
\end{array}\right]
$$

with $x \in \mathbb{R}$ and $j \geq 1$, and the block vector $v_{j, 2 j-1}=\left[\mu_{j} \mu_{j+1} \cdots \mu_{2 j-1}\right]^{H} \in \mathcal{M}_{l j \times l}$. Notice that $H_{j}$ is a Hankel invertible and Hermitian matrix for $j \geq 1$. With these matrices, it is shown in [27] that the sequence of (right) matrix polynomials $\left(P_{j}^{R}(x)\right)_{j \geq 0}$ defined by

$$
P_{j}^{R}(x)=x^{j} \mathbf{I}-\left[\mathbf{I} x \mathbf{I} \cdots x^{j-1} \mathbf{I}\right] H_{j}^{-1} v_{j, 2 j-1}, \quad \text { with } P_{0}^{R}(x)=\mathbf{I}
$$

(i.e., each $P_{j}^{R}$ is defined as the Schur complement of $x^{j} \mathbf{I}$ in the matrix $F_{j}$ ), is orthogonal with respect to the sesquilinear form 2.2 , that is,

$$
\left\langle P_{m}^{R}, P_{j}^{R}\right\rangle_{R, \mu}=\delta_{m, j} s_{j}, \quad m=0, \ldots, j,
$$

where $s_{j}$ is the Schur complement of $H_{j+1}$ with respect to $\mu_{2 j}$, i.e.

$$
s_{j}=\mu_{2 j}-v_{j, 2 j-1}^{H} H_{j}^{-1} v_{j, 2 j-1}, \quad \text { with } s_{0}=\mu_{0} .
$$

Conversely, any sequence of matrix moments $\left(\mu_{j}\right)_{j \geq 0}$ satisfying that the corresponding Hankel matrices are invertible and Hermitian defines a Hermitian matrix measure. In this situation, we will say $\left(\mu_{j}\right)_{j \geq 0}$ is a Hermitian sequence. The positiveness of $\mu$ can also be characterized in terms of the norms $s_{j}, j \geq 0$, as follows. 
Theorem 2.1 ([27]). Let $\mu$ be a matrix measure supported on $E \subseteq \mathbb{R}$. Then, $\mu$ is positive semi-definite for every $x \in E$ if and only if $s_{j}$ is positive semi-definite for every $j \geq 0$.

In a similar way, a sequence of left matrix polynomials $\left(P_{n}^{L}(x)\right)_{n \geq 0}$ can be defined such that it is orthogonal with respect to the sesquilinear form 2.3$)$. Since $P_{j}^{L}(x)=$ $P_{j}^{R}(x)^{H}$ for all $x \in \mathbb{R}$, it suffices to consider $P_{j}(x)=P_{j}^{R}(x)$.

Let $P_{j}^{(k)}(x)$ be the $k$-th derivative of $P_{j}(x)$. From 2.4 we can deduce

$$
\frac{P_{j}^{(k)}(0)}{k !}= \begin{cases}-\left(\chi_{k}^{(j)}\right)^{H} H_{j}^{-1} v_{j, 2 j-1}, & \text { if } 0 \leq k \leq j-1 \\ \mathbf{I}, & \text { if } k=j\end{cases}
$$

The kernel matrix polynomial of degree $j$ for $x, y \in \mathbb{R}$ is defined by

$$
K_{j}(y, x)=\sum_{k=0}^{j} P_{k}(x) s_{k}^{-1} P_{k}(y)^{H},
$$

which can be expressed also in terms of the moments by (see [27])

$$
K_{j}(y, x)=\left[\mathbf{I} x \mathbf{I} \cdots x^{j} \mathbf{I}\right] H_{j+1}^{-1}\left[\mathbf{I} y \mathbf{I} \cdots y^{j} \mathbf{I}\right]^{T} .
$$

Denoting $K_{j}^{(i, r)}(y, x)$ the $i$-th (resp. $r$-th) derivative of $K_{j}(y, x)$ with respect to the variable $y(\operatorname{resp} . x)$, we have from $(2.7)$, for $0 \leq i \leq j$ and $0 \leq r \leq j$,

$$
\begin{aligned}
& \frac{K_{j}^{(i, 0)}(0, x)}{i !}=\left[\mathbf{I} x \mathbf{I} \cdots x^{j} \mathbf{I}\right] H_{j+1}^{-1} \chi_{i}^{(j+1)}, \\
& \frac{K_{j}^{(0, r)}(y, 0)}{r !}=\left(\chi_{r}^{(j+1)}\right)^{H} H_{j+1}^{-1}\left[\mathbf{I} y \mathbf{I} \cdots y^{j} \mathbf{I}\right]^{T}, \\
& \frac{K_{j}^{(i, r)}(0,0)}{i ! r !}=\left(\chi_{r}^{(j+1)}\right)^{H} H_{j+1}^{-1} \chi_{i}^{(j+1)} .
\end{aligned}
$$

2.2. Matrix orthogonal polynomials on the unit circle. Let $\mathbb{T}:=\{z \in \mathbb{C}$ : $|z|=1\}$ and $\mathbb{D}:=\{z \in \mathbb{C}:|z|<1\}$ be the unit circle and unit disc, respectively, where $z \in \mathbb{T}$ is parametrized as $z=e^{i \theta}$ with $\theta \in(-\pi, \pi]$. In what follows, $\sigma=$ $\left(\sigma_{i, j}\right)_{i, j=1}^{l}$ will be a Hermitian matrix measure supported on $\mathbb{T}$ (on all or part of $\mathbb{T})$ and $\mathbb{P}^{l}[z]$ will denote the linear space of polynomials in $z \in \mathbb{C}$ with coefficients in $\mathcal{M}_{l}$. A Hermitian matrix measure $\sigma$ can be decomposed as

$$
d \sigma(\theta)=\mathcal{W}(\theta) \frac{d \theta}{2 \pi}+d \sigma_{s}(\theta)
$$

where the Hermitian matrix $\mathcal{W}(\theta)$ denotes the Radon-Nikodym derivative of the absolutely continuous part of $\sigma$ with respect to the scalar Lebesgue measure, and $\sigma_{s}$ represents the singular part. 
As in the real line case, a matrix measure $\sigma$ induces right and left sesquilinear forms, defined respectively as

$$
\begin{aligned}
& \langle p, q\rangle_{R, \sigma}=\int_{-\pi}^{\pi} p\left(e^{i \theta}\right)^{H} d \sigma(\theta) q\left(e^{i \theta}\right) \in \mathcal{M}_{l}, \quad p, q \in \mathbb{P}^{l}[z], \\
& \langle p, q\rangle_{L, \sigma}=\int_{-\pi}^{\pi} p\left(e^{i \theta}\right) d \sigma(\theta) q\left(e^{i \theta}\right)^{H} \in \mathcal{M}_{l}, \quad p, q \in \mathbb{P}^{l}[z] .
\end{aligned}
$$

On the other hand, the $j$-th (matrix) moment associated with the matrix measure $\sigma$ is

$$
c_{j}=\left\langle\mathbf{I}, z^{j} \mathbf{I}\right\rangle_{R, \sigma}=\left\langle z^{j} \mathbf{I}, \mathbf{I}\right\rangle_{L, \sigma}=\int_{-\pi}^{\pi} e^{i j \theta} d \sigma(\theta) \in \mathcal{M}_{l}, \quad j \in \mathbb{Z}, j \neq 0,
$$

and $c_{0}+c_{0}^{H}:=\int_{-\pi}^{\pi} d \sigma(\theta)$. Notice that every matrix moment satisfies $c_{-j}^{H}=c_{j}$ for $j \in \mathbb{Z}$, since $\sigma$ is an Hermitian measure. Let $T_{j} \in \mathcal{M}_{l j}$ and $M_{j} \in \mathcal{M}_{l(j+1)}$ be defined as

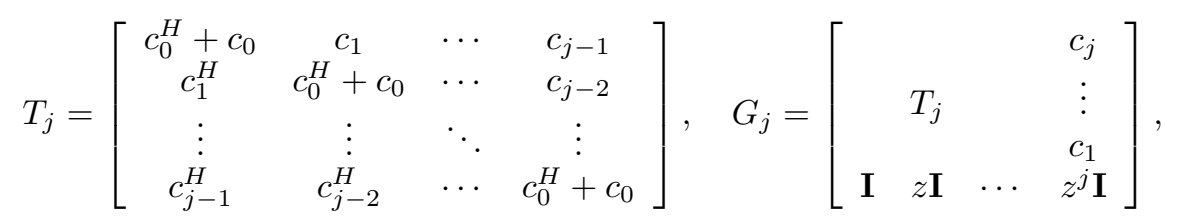

and the vector block $\nu_{j}=\left[\begin{array}{llll}c_{j}^{H} & c_{j-1}^{H} \cdots & c_{1}^{H}\end{array}\right]^{H} \in \mathcal{M}_{l j \times l}$. Notice that $T_{j}$ is a Toeplitz invertible and Hermitian matrix for $j \geq 1$. As in the real line case, any sequence $\left(c_{j}\right)_{j \in \mathbb{Z}}$ such that $T_{j}$ is invertible and Hermitian for $j \geq 1$ will be called a Hermitian sequence, and has an associated Hermitian matrix measure $\sigma$ supported on $\mathbb{T}$. The sequence of monic matrix polynomials $\left(\Phi_{j}^{R}(z)\right)_{j \geq 0}$ defined by (see [26])

$$
\Phi_{j}^{R}(z)=z^{j} \mathbf{I}-\left[\mathbf{I} z \mathbf{I} \cdots z^{j-1} \mathbf{I}\right] T_{j}^{-1} \nu_{j}, \quad \text { with } \Phi_{0}^{R}(z)=\mathbf{I},
$$

is orthogonal with respect to the sesquilinear form 2.8 and satisfies

$$
\left\langle\Phi_{m}^{R}, \Phi_{j}^{R}\right\rangle_{R, \sigma}=\delta_{m, j} S_{j}^{R}, \quad m=0, \ldots, j,
$$

where the Hermitian matrix $S_{j}^{R}$ is the Schur complement of $T_{j+1}$ with respect to $c_{0}+c_{0}^{H}$, i.e.,

$$
S_{j}^{R}=c_{0}^{H}+c_{0}-\nu_{j}^{H} T_{j}^{-1} \nu_{j}, \quad \text { with } S_{0}^{R}=c_{0}+c_{0}^{H} .
$$

In a similar way, a sequence $\left(\Phi_{n}^{L}(z)\right)_{n \geq 0}$ can be constructed such that it is orthogonal with respect to the sesquilinear form (2.9). Unlike the real line case, where the right-orthogonal matrix polynomial is just the conjugate transpose of the left-orthogonal matrix polynomial, on the unit circle there is not a direct relation between the right and left-orthogonal polynomials. This is a consequence of the fact that $\langle z f, g\rangle_{R, \sigma}=\langle f, \bar{z} g\rangle_{R, \sigma}$. Nevertheless, we will consider only the right matrix polynomials, denoted by $\Phi_{j}=\Phi_{j}^{R}$ and the corresponding norms $S_{j}=S_{j}^{R}$ for $j \geq 0$, since the results for the left polynomials are analogous. 
Let $\Phi_{j}^{(k)}(x)$ be the $k$-th derivative of $\Phi_{j}(x)$. From 2.10 we can deduce

$$
\frac{\Phi_{j}^{(k)}(0)}{k !}= \begin{cases}-\left(\chi_{k}^{(j)}\right)^{H} T_{j}^{-1} \nu_{j}, & \text { if } 0 \leq k \leq j-1, \\ \mathbf{I}, & \text { if } k=j .\end{cases}
$$

Theorem 2.1 is valid also for the matrix measure $\sigma$ with support on $\mathbb{T}$. The kernel matrix polynomial of degree $j$ for $z, w \in \mathbb{C}$ is defined in this case by

$$
K_{j}(w, z)=\sum_{k=0}^{j} \Phi_{k}(z) S_{k}^{-1} \Phi_{k}(w)^{H}
$$

and its corresponding expression in terms of the matrix moments is (see [26])

$$
K_{j}(w, z)=\left[\mathbf{I} z \mathbf{I} \cdots z^{j} \mathbf{I}\right] T_{j+1}^{-1}\left[\mathbf{I} \bar{w} \mathbf{I} \cdots \bar{w}^{j} \mathbf{I}\right]^{T} .
$$

As in the real case, $K_{j}^{(i, r)}(w, z)$ is the $i$-th (resp. $r$-th) derivative of $K_{j}(w, z)$ with respect to the variable $w$ (resp. $z$ ). Thus, from (2.13), for $0 \leq i \leq j$ and $0 \leq r \leq j$, we have

$$
\begin{aligned}
\frac{K_{j}^{(i, 0)}(0, z)}{i !} & =\left[\mathbf{I} x \mathbf{I} \cdots z^{j} \mathbf{I}\right] T_{j+1}^{-1} \chi_{i}^{(j+1)}, \\
\frac{K_{j}^{(0, r)}(w, 0)}{r !} & =\left(\chi_{r}^{(j+1)}\right)^{H} T_{j+1}^{-1}\left[\mathbf{I} \bar{w} \mathbf{I} \cdots \bar{w}^{j} \mathbf{I}\right]^{T}, \\
\frac{K_{j}^{(i, r)}(0,0)}{i ! r !} & =\left(\chi_{r}^{(j+1)}\right)^{H} T_{j+1}^{-1} \chi_{i}^{(j+1)} .
\end{aligned}
$$

\section{Matrix moments PeRturbation AND its CONNECTiOn THROUGH THE INVERSE SZEGŐ TRANSFORMATION}

In this section, we define the Szegö matrix transformation of a matrix measure supported on the real line, as well as some of its properties. Later on, we will define a perturbation of a matrix measure supported on $\mathbb{T}$ that consists in the perturbation of a single matrix moment of such measure, and analyze the resulting perturbation on the real line when the measures are related through the Szego" matrix transformation.

3.1. Szegő matrix transformation. A matrix measure $\sigma(\theta)$ supported on $\mathbb{T}$ that is invariant under $\theta \mapsto-\theta$ (i.e., $z \mapsto \bar{z}=z^{-1}$ ) is called symmetric. If $\sigma(\theta)$ is symmetric and positive semi-definite, then its associated matrix moments are Hermitian matrices, since

$$
c_{n}=\int_{-\pi}^{\pi} e^{i n \theta} d \sigma(\theta)=\int_{-\pi}^{\pi} e^{-i n \theta} d \sigma(\theta)=c_{-n}=c_{n}^{H}, \quad \text { for } n \in \mathbb{Z} .
$$

On the other hand, if $\mu(x)$ is a positive semi-definite matrix measure supported on $[-1,1]$, it is possible to define a symmetric positive semi-definite matrix measure 
$\sigma(\theta)$ supported on $(-\pi, \pi]$ by

$$
d \sigma(\theta)= \begin{cases}\frac{1}{2} d \mu(\cos \theta), & -\pi \leq \theta \leq 0 \\ -\frac{1}{2} d \mu(\cos \theta), & 0 \leq \theta \leq \pi\end{cases}
$$

This is the so-called Szegö matrix transformation (see [8, 9, 33]). The scalar version of the Szegö transformation can be found in [30]. We will write $d \sigma=S z(d \mu)$ if the matrix measures $\mu$ and $\sigma$ satisfy

$$
\int_{-1}^{1} f(x) d \mu(x)=\int_{-\pi}^{\pi} f(\cos \theta) d \sigma(\theta)
$$

for any integrable function $f$ on $[-1,1]$. In a similar way, the inverse Szegö matrix transformation can be defined by the relation

$$
\int_{-\pi}^{\pi} g(\theta) d \sigma(\theta)=\int_{-1}^{1} g(\arccos x) d \mu(x)
$$

for any integrable function $g$ on $\mathbb{T}$ such that $g(\theta)=g(-\theta)$. We will denote this relation by $d \mu=S z^{-1}(d \sigma)$. Notice that if $\mu$ has the form (2.1), then (3.1) becomes

$$
d \sigma(\theta)= \begin{cases}-\frac{1}{2} \omega(\cos \theta) \sin \theta d \theta, & -\pi \leq \theta \leq 0, \\ \frac{1}{2} \omega(\cos \theta) \sin \theta d \theta, & 0 \leq \theta \leq \pi,\end{cases}
$$

which can be written as

$$
d \sigma(\theta)=\frac{1}{2}|\sin \theta| \omega(\cos \theta) d \theta
$$

If $d \mu(x)=\omega(x) d x$, and $d \sigma(\theta)=\mathcal{W}(\theta) \frac{d \theta}{2 \pi}$, then they are related through the Szego" matrix transformation when

$$
\begin{gathered}
\mathcal{W}(\theta)=\pi|\sin \theta| \omega(\cos \theta), \quad \omega(x)=\frac{1}{\pi} \frac{1}{\sqrt{1-x^{2}}} \mathcal{W}(\arccos x), \\
S z\left(\frac{1}{\pi} \frac{1}{\sqrt{1-x^{2}}} \mathcal{W}(\arccos x) d x\right)=\pi|\sin \theta| \omega(\cos \theta) \frac{d \theta}{2 \pi} .
\end{gathered}
$$

In particular, for $\mathcal{W}(\theta)=\cos ^{k} \theta$, with $k \geq 0$, we have

$$
S z^{-1}\left(\cos ^{k} \theta \frac{d \theta}{2 \pi}\right)=\frac{1}{\pi} \frac{x^{k}}{\sqrt{1-x^{2}}} d x .
$$

The proof is analogous to the one given in [30, Proposition 13.1.1].

3.2. Matrix moments on real line and the unit circle related through the inverse Szegő transformation. Let $\langle\cdot, \cdot\rangle_{R, \theta}$ be a right sesquilinear form defined by

$$
\langle p, q\rangle_{R, \theta}=\int_{-\pi}^{\pi} p\left(e^{i \theta}\right)^{H} q\left(e^{i \theta}\right) \frac{d \theta}{2 \pi}, \quad p, q \in \mathbb{P}^{l}[z] .
$$


Given a positive semi-definite matrix measure $\sigma$ supported on $\mathbb{T}$, we can consider the perturbation

$$
d \sigma_{j}(\theta)=d \sigma(\theta)+z^{j} m_{j} \frac{d \theta}{2 \pi}+z^{-j} m_{j}^{H} \frac{d \theta}{2 \pi}, \quad z=e^{i \theta},
$$

for a fixed $j$ and $m_{j} \in \mathcal{M}_{l}$ such that $\sigma_{j}$ is positive semi-definite. In particular, if $\sigma$ is symmetric and $m_{j}$ is a Hermitian matrix, then 3.5 becomes

$$
d \sigma_{j}(\theta)=d \sigma(\theta)+2 \cos (j \theta) m_{j} \frac{d \theta}{2 \pi}
$$

and thus $\sigma_{j}$ is a symmetric, positive semi-definite matrix measure supported on the unit circle.

Notice that the right sesquilinear form associated with 3.5$)$ is

$$
\langle p, q\rangle_{R, \sigma_{j}}=\langle p, q\rangle_{R, \sigma}+\left\langle m_{j}^{H} p, z^{j} q\right\rangle_{R, \theta}+\left\langle z^{j} m_{j} p, q \cdot\right\rangle_{R, \theta}, \quad p, q \in \mathbb{P}^{l}[z] .
$$

From $(3.7)$, one easily sees that the corresponding matrix moment $\tilde{c}_{k}$ satisfies

$$
\tilde{c}_{k}= \begin{cases}c_{k}, & \text { if } k \notin\{j,-j\}, \\ c_{j}+m_{j}^{H}, & \text { if } k=j, \\ c_{-j}+m_{j}, & \text { if } k=-j .\end{cases}
$$

In other words, $\sigma_{j}$ represents an additive perturbation of the matrix moments $c_{j}$ and $c_{-j}$ of $\sigma$.

The following result shows the matrix measure supported on $[-1,1]$ that is related with (3.5) through the inverse Szegö matrix transformation. This result constitutes a generalization of the corresponding result on the scalar case studied in $[18,19]$.

Proposition 3.1. Let $\sigma$ be a positive semi-definite matrix measure supported on $\mathbb{T}$, and let $m_{j} \in \mathcal{M}_{l}$ be a Hermitian matrix such that $\sigma_{j}$ defined as in (3.5) is positive semi-definite. Assume $d \mu=S z^{-1}(d \sigma)$. Then, $d \mu_{j}=S z^{-1}\left(d \sigma_{j}\right)$ is given by

$$
d \mu_{j}(x)=d \mu(x)+2 T_{j}(x) m_{j} \frac{d x}{\pi \sqrt{1-x^{2}}}, \quad j \geq 0,
$$

where $T_{j}(x):=\cos (j \theta)$ is the $j$-th degree Chebyshev polynomial of the first kind on $\mathbb{R}$ (see [5]).

Proof. Let $T_{j}(x)=\sum_{k=0}^{j} a_{k} x^{k}, x=\cos \theta, z=e^{i \theta}=\cos \theta+i \sin \theta$, and $m_{j}$ an Hermitian matrix. Then, from 3.5 ,

$$
\begin{aligned}
d \sigma_{j}(\theta) & =d \sigma(\theta)+(\cos \theta+i \sin \theta)^{j} m_{j} \frac{d \theta}{2 \pi}+(\cos \theta+i \sin \theta)^{-j} m_{j} \frac{d \theta}{2 \pi} \\
& =d \sigma(\theta)+2 T_{j}(x) m_{j} \frac{d \theta}{2 \pi} \\
& =d \sigma(\theta)+2 \sum_{k=0}^{j} a_{k} \cos ^{k} \theta m_{j} \frac{d \theta}{2 \pi} .
\end{aligned}
$$


Since $\sigma_{j}(\theta)$ is a symmetric matrix measure, by applying the inverse Szegő matrix transformation and taking into account (3.4), we have

$$
\begin{aligned}
d \mu_{j}(x) & =d \mu(x)+2 \sum_{k=0}^{j} a_{k} x^{k} m_{j} \frac{d x}{\pi \sqrt{1-x^{2}}} \\
& =d \mu(x)+2 T_{j}(x) m_{j} \frac{d x}{\pi \sqrt{1-x^{2}}}
\end{aligned}
$$

which is 3.8 .

Recall that the double factorial or semifactorial of a number $n$ (denoted by $n !$ !) is the product of all the integers from 1 up to $n$ that have the same parity (odd or even) as $n$. That is,

$$
n ! != \begin{cases}\prod_{k=1}^{n / 2}(2 k)=n(n-2)(n-4) \cdots(4)(2), & \text { if } n \text { is even, } \\ \prod_{k=1}^{(n+1) / 2}(2 k-1)=n(n-2)(n-4) \cdots(3)(1), & \text { if } n \text { is odd } .\end{cases}
$$

The next proposition expresses the considered perturbation in terms of the moments associated with the matrix measure 3.8.

Proposition 3.2. Let $\mu$ be a positive semi-definite matrix measure supported on $[-1,1], \mu_{j}$ defined as in (3.8), and let $\left(\mu_{n}\right)_{n \geq 0},\left(\tilde{\mu}_{n}\right)_{n \geq 0}$ be their corresponding sequences of matrix moments. Then,

$$
\tilde{\mu}_{n}= \begin{cases}\mu_{n}+m_{j} \mathbb{B}(n, j), & \text { if } j \leq n \text { and } n+j \text { is even }, \\ \mu_{n}, & \text { otherwise }\end{cases}
$$

where

$$
\mathbb{B}(n, j)=j \sum_{k=0}^{[j / 2]}(-1)^{k} \frac{(j-k-1) !(2)^{j-2 k}}{k !(j-2 k) !} \frac{(j+n-2 k-1) ! !}{(j+n-2 k) ! !} .
$$

Proof. Since $T_{j}(x)$ is orthogonal with respect to $\frac{1}{\sqrt{1-x^{2}}}$, we have

$$
\tilde{\mu}_{n}=\int_{-1}^{1} x^{n} d \tilde{\mu}_{j}(x)= \begin{cases}\mu_{n}+\frac{2}{\pi} m_{j} \int_{-1}^{1} x^{n} T_{j}(x) \frac{d x}{\sqrt{1-x^{2}}}, & \text { if } j \leq n, \\ \mu_{n}, & \text { otherwise. }\end{cases}
$$

Moreover, from the symmetry, $\int_{-1}^{1} x^{n} T_{j}(x) \frac{d x}{\sqrt{1-x^{2}}}=0$ if $n+j$ is odd, and thus

$$
\tilde{\mu}_{n}= \begin{cases}\mu_{n}+\frac{2}{\pi} m_{j} \int_{-1}^{1} x^{n} T_{j}(x) \frac{d x}{\sqrt{1-x^{2}}}, & \text { if } j \leq n \text { and } n+j \text { is even, } \\ \mu_{n}, & \text { otherwise. }\end{cases}
$$

Furthermore (see [23]), we have

$$
T_{j}(x)=\frac{j}{2} \sum_{k=0}^{[j / 2]} \frac{(-1)^{k}(j-k-1) !(2 x)^{j-2 k}}{k !(j-2 k) !}, \quad j \geq 1,
$$


where $[j / 2]=j / 2$ if $j$ is even and $[j / 2]=(j-1) / 2$ if $j$ is odd. As a consequence, if $j \leq n$ and $n+j$ is even,

$$
\begin{aligned}
\int_{-1}^{1} x^{n} T_{j}(x) \frac{d x}{\sqrt{1-x^{2}}} & =\frac{j}{2} \int_{-1}^{1} x^{n} \sum_{k=0}^{[j / 2]} \frac{(-1)^{k}(j-k-1) !(2 x)^{j-2 k}}{k !(j-2 k) !} \frac{d x}{\sqrt{1-x^{2}}} \\
& =\frac{j}{2} \sum_{k=0}^{[j / 2]} \frac{(-1)^{k}(j-k-1) !(2)^{j-2 k}}{k !(j-2 k) !} \int_{-1}^{1} x^{j+n-2 k} \frac{d x}{\sqrt{1-x^{2}}}
\end{aligned}
$$

and, since

$$
\int_{-1}^{1} x^{n} \frac{d x}{\sqrt{1-x^{2}}}=\pi \frac{(n-1) ! !}{n ! !}
$$

for $n$ even, and equals zero if $n$ is odd, 3.10 becomes 3.9 .

This means that the perturbation of the matrix moments $c_{j}$ and $c_{-j}$ of a matrix measure $\sigma$ supported on the unit circle results in a perturbation, defined by $(3.9)$, of the moments $\mu_{n}$ associated with a measure $\mu$ supported in $[-1,1]$, when both measures are related through the inverse matrix Szegő transformation.

\section{Connection formulas on the Real Line And Further Results}

Let $\mu$ be a Hermitian matrix measure supported on $E \subseteq \mathbb{R}$ with an associated sequence of monic matrix orthogonal polynomials $\left(P_{n}(x)\right)_{n \geq 0}$, and a Hermitian sequence of matrix moments $\left(\mu_{n}\right)_{n \geq 0}$. Define a new matrix moments sequence $\left(\tilde{\mu}_{n}\right)_{n \geq 0}$ by $\tilde{\mu}_{n}=\mu_{n}+\mathbf{M}_{n} \in \mathcal{M}_{l}$ with $\mathbf{M}_{n}$ Hermitian, in such a way that $\left(\tilde{\mu}_{n}\right)_{n \geq 0}$ is also a Hermitian sequence. Then, there exists an associated measure $\tilde{\mu}$ that is Hermitian, with a corresponding sequence of matrix orthogonal polynomials $\left(\tilde{P}_{n}(x)\right)_{n \geq 0}$. Also, define

$$
s_{j}=\left\langle P_{j}, P_{j}\right\rangle_{R, \mu} \quad \text { and } \quad \tilde{s}_{j}=\left\langle\tilde{P}_{j}, \tilde{P}_{j}\right\rangle_{R, \tilde{\mu}}
$$

In this section, we will show the relation between the matrix polynomials $\tilde{P}_{j}$ and $P_{j}$, as well as the relation between $\tilde{s}_{j}$ and $s_{j}$, in terms of the sequence of matrix moments $\left(\mu_{n}\right)_{n \geq 0}$. Notice that the perturbation of a matrix measure $\mu$ supported on $E=[-1,1]$ defined by 3.8 constitutes a particular case with

$$
\mathbf{M}_{n}= \begin{cases}m_{j} \mathbb{B}(n, j), & \text { if } j \leq n \text { and } n+j \text { is even } \\ \mathbf{0}, & \text { otherwise. }\end{cases}
$$


We will use the following notation:

$$
\begin{aligned}
\mathbf{K}_{j-1}^{k}(0, x) & :=\left[K_{j-1}^{(k, 0)}(0, x), K_{j-1}^{(k-1,0)}(0, x), \ldots, K_{j-1}^{(0,0)}(0, x)\right] \in \mathcal{M}_{l \times(k+1) l}, \\
\mathbf{P}_{j, k}(0) & :=\left[P_{j}^{(0)}(0)^{T}, P_{j}^{(1)}(0)^{T}, \ldots, P_{j}^{(k)}(0)^{T}\right]^{T} \in \mathcal{M}_{(k+1) l \times l}, \\
\tilde{\mathbf{P}}_{j, k}(0) & :=\left[\tilde{P}_{j}^{(0)}(0)^{T}, \tilde{P}_{j}^{(1)}(0)^{T}, \ldots, \tilde{P}_{j}^{(k)}(0)^{T}\right]^{T} \in \mathcal{M}_{(k+1) l \times l}, \\
\mathbf{D}_{0, k} & :=\operatorname{diag}\{0 ! \mathbf{I}, 1 ! \mathbf{I}, \ldots, k ! \mathbf{I}\} \in \mathcal{M}_{(k+1) l}, \\
\mathbf{D}_{k, 0} & :=\operatorname{diag}\{k ! \mathbf{I},(k-1) ! \mathbf{I}, \ldots, 0 ! \mathbf{I}\} \in \mathcal{M}_{(k+1) l}, \\
\mathbf{D}_{\mathbf{M}_{k}} & :=\operatorname{diag}\left\{\mathbf{M}_{k}, \ldots, \mathbf{M}_{k}\right\} \in \mathcal{M}_{(k+1) l} .
\end{aligned}
$$

For a fixed $k$, with $0 \leq k \leq 2 j-1$, it has been proved in [7] that

$$
\begin{aligned}
\tilde{P}_{j}(x) & =P_{j}(x)-\sum_{p=0}^{k} \frac{K_{j-1}^{(k-p, 0)}(0, x)}{(k-p) !} \mathbf{M}_{k} \frac{\tilde{P}_{j}^{(p)}(0)}{p !} \\
& =P_{j}(x)-\mathbf{K}_{j-1}^{k}(0, x) \mathbf{D}_{k, 0}^{-1} \mathbf{D}_{\mathbf{M}_{k}} \mathbf{D}_{0, k}^{-1} \tilde{\mathbf{P}}_{j, k}(0),
\end{aligned}
$$

with

$$
\tilde{\mathbf{P}}_{j, k}(0)=\left[\mathbf{I}_{(k+1) l}+\mathbf{D}_{0, k}\left(\Lambda_{0, k}^{(j)}\right)^{H} H_{j}^{-1} \Lambda_{0, k}^{(j)} \mathbf{D}_{k, 0}^{-1} \mathbf{D}_{\mathbf{M}_{k}} \Lambda_{k, 0}^{(k+1)}\right]^{-1} \mathbf{P}_{j, k}(0) .
$$

As a consequence we have the next result.

Theorem 4.1 ([7]). Let $\mu$ and $\tilde{\mu}$ be Hermitian matrix measures associated with the Hermitian sequences $\left(\mu_{k}\right)_{k=0}^{2 n-1}$ and $\left(\tilde{\mu}_{k}\right)_{k=0}^{2 n-1}$, respectively, where $\tilde{\mu}_{k}=\mu_{k}+\boldsymbol{M}_{k}$, with $0 \leq k \leq 2 n-1$. For $1 \leq j \leq n,\left(\tilde{P}_{j}(x)\right)_{j=0}^{n}$ is orthogonal with respect to $\tilde{\mu}$ if

$$
\tilde{P}_{j}(x)=P_{j}(x)-\sum_{k=0}^{2 n-1} \boldsymbol{K}_{j-1}^{k}(0, x) \mathbb{M}(k, j) \boldsymbol{P}_{j, k}(0), \quad \text { with } \tilde{P}_{0}(x)=P_{0}(x)=\boldsymbol{I},
$$

where

$$
\mathbb{M}(k, j)=\boldsymbol{D}_{k, 0}^{-1} \boldsymbol{D}_{\boldsymbol{M}_{k}} \boldsymbol{D}_{0, k}^{-1}\left[\boldsymbol{I}_{(k+1) l}+\boldsymbol{D}_{0, k}\left(\Lambda_{0, k}^{(j)}\right)^{H} H_{j}^{-1} \Lambda_{0, k}^{(j)} \boldsymbol{D}_{k, 0}^{-1} \boldsymbol{D}_{\boldsymbol{M}_{k}} \Lambda_{k, 0}^{(k+1)}\right]^{-1} .
$$

Now, we will deduce a relation for the corresponding norms. We will need the following auxiliary results. For $0 \leq j \leq n$, let $\tilde{\mathbf{M}}_{j} \in \mathcal{M}_{j l}$ be defined as

$$
\tilde{\mathbf{M}}_{j}:=\left[\begin{array}{ccccc}
\mathbf{M}_{0} & \cdots & \mathbf{M}_{k} & \cdots & \mathbf{M}_{j-1} \\
\vdots & \mathbf{M}_{k} & & & \vdots \\
\mathbf{M}_{k} & & \vdots & & \mathbf{M}_{2 j-2-k} \\
\vdots & & & \mathbf{M}_{2 j-2-k} & \vdots \\
\mathbf{M}_{j-1} & \cdots & \mathbf{M}_{2 j-2-k} & \cdots & \mathbf{M}_{2 j-2}
\end{array}\right]=\sum_{k=0}^{j-1} \mathbf{M}_{j, k}+\sum_{k=j}^{2 j-2} \mathbf{M}_{j, k}^{t}
$$


where for $k=0, \ldots, j-1$ (resp. $k=j, \ldots, 2 j-2$ ), the matrices $\mathbf{M}_{j, k} \in \mathcal{M}_{j l}$ (resp. $\left.\mathbf{M}_{j, k}^{t} \in \mathcal{M}_{j l}\right)$ are given by

$\mathbf{M}_{j, k}:=\left[\begin{array}{ccccc}\mathbf{0} & \cdots & \mathbf{M}_{k} & \cdots & \mathbf{0} \\ \vdots & \mathbf{M}_{k} & & & \vdots \\ \mathbf{M}_{k} & & \vdots & & \vdots \\ \vdots & & & \ddots & \\ \mathbf{0} & \cdots & & \cdots & \mathbf{0}\end{array}\right], \quad \mathbf{M}_{j, k}^{t}:=\left[\begin{array}{ccccc}\mathbf{0} & \cdots & & \cdots & \mathbf{0} \\ \vdots & \ddots & & & \vdots \\ \vdots & & \vdots & & \mathbf{M}_{k} \\ \vdots & & & \mathbf{M}_{k} & \vdots \\ \mathbf{0} & \cdots & \mathbf{M}_{k} & \cdots & \mathbf{0}\end{array}\right]$,

and for $k \geq 2 j-1, \mathbf{M}_{j, k}=\mathbf{M}_{j, k}^{t}=\mathbf{0} \in \mathcal{M}_{j l}$.

Lemma 4.2. Let

$$
\tilde{H}_{j, k}= \begin{cases}H_{j}+\boldsymbol{M}_{j, k}, & \text { if } k=0, \ldots, j-1 \\ H_{j}+\boldsymbol{M}_{j, k}^{t}, & \text { if } k=j, \ldots, 2 j-2 .\end{cases}
$$

(1) If $k=0, \ldots, j-1$, then

$$
\tilde{H}_{j, k}^{-1}-H_{j}^{-1}=-\sum_{p=0}^{k} H_{j}^{-1} \chi_{k-p}^{(j)} \boldsymbol{M}_{k}\left(\chi_{p}^{(j)}\right)^{H} \tilde{H}_{j, k}^{-1} .
$$

(2) If $k=j, \ldots, 2 j-2$, then

$$
\tilde{H}_{j, k}^{-1}-H_{j}^{-1}=-\sum_{p=k-(j-1)}^{j-1} H_{j}^{-1} \chi_{k-p}^{(j)} \boldsymbol{M}_{k}\left(\chi_{p}^{(j)}\right)^{H} \tilde{H}_{j, k}^{-1} .
$$

Proof. For $k=0, \ldots, j-1$, we have

$$
\tilde{H}_{j, k}^{-1}-H_{j}^{-1}=H_{j}^{-1}\left(H_{j}-\tilde{H}_{j, k}\right) \tilde{H}_{j, k}^{-1}=-H_{j}^{-1} \mathbf{M}_{j, k} \tilde{H}_{j, k}^{-1},
$$

and thus

$$
\mathbf{M}_{j, k}=\chi_{k}^{(j)} \mathbf{M}_{k}\left(\chi_{0}^{(j)}\right)^{H}+\cdots+\chi_{0}^{(j)} \mathbf{M}_{k}\left(\chi_{k}^{(j)}\right)^{H}=\sum_{p=0}^{k} \chi_{k-p}^{(j)} \mathbf{M}_{k}\left(\chi_{p}^{(j)}\right)^{H} .
$$

Substituting $\mathbf{M}_{j, k}$ in (4.7), 4.5 follows. A similar procedure can be used to prove (4.6).

Let $\tilde{H}_{j} \in \mathcal{M}_{l j}$ be the block Hankel matrix associated with the perturbed matrix moments sequence, i.e.

$$
\tilde{H}_{j}=\left[\begin{array}{cccc}
\tilde{\mu_{0}} & \tilde{\mu_{1}} & \cdots & \mu_{\tilde{j}-1} \\
\tilde{\mu_{1}} & \tilde{\mu_{2}} & \cdots & \tilde{\mu_{j}} \\
\vdots & \vdots & \ddots & \vdots \\
\mu_{\tilde{j}-1} & \tilde{\mu_{j}} & \cdots & \mu_{2 \tilde{j}-2}
\end{array}\right]=H_{j}+\tilde{\mathbf{M}}_{j} .
$$

Thus, from 4.4 for $1 \leq j \leq n$,

$$
\tilde{H}_{j}=\tilde{H}_{j, 0}+\cdots+\tilde{H}_{j, 2 j-2} .
$$


Theorem 4.3. Let $\mu$ be a Hermitian matrix measure with matrix moments $\left(\mu_{k}\right)_{k=0}^{2 n-1}$. Define a new Hermitian sequence of matrix moments $\left(\tilde{\mu}_{k}\right)_{k=0}^{2 n-1}$ by $\tilde{\mu}_{k}=\mu_{k}+\mathbf{M}_{k}$ with $0 \leq k \leq 2 n-1$, and denote by $\tilde{\mu}$ its corresponding Hermitian matrix measure. Then, for $1 \leq j \leq n$, we have

$$
\tilde{s}_{j}=s_{j}+\sum_{k=0}^{2 n-1} \boldsymbol{P}_{j, k}(0)^{H} \Lambda_{k, 0}^{(k+1)} \mathbb{M}(k, j) \boldsymbol{P}_{j, k}(0),
$$

with $\tilde{s}_{0}=\tilde{\mu}_{0}=\mu_{0}+\mathbf{M}_{0}$ and $\mathbb{M}(k, j)$ as in 4.3 .

Proof. Let us assume first that only the $k$-th moment is perturbed. From 2.5 we have that if $2 j<k$, then $\tilde{s}_{j}=s_{j}$. We will show that if $k \leq 2 j$, we have

$$
\tilde{s}_{j}=s_{j}+\sum_{p=0}^{k} \frac{\left(P_{j}^{(k-p)}(0)\right)^{H}}{(k-p) !} \mathbf{M}_{k} \frac{\tilde{P}_{j}^{(p)}(0)}{p !} .
$$

For $0 \leq k \leq j-1$, notice that

$$
\tilde{\mu}_{2 j}=\mu_{2 j} \quad \text { and } \quad \tilde{v}_{j, 2 j-1}=v_{j, 2 j-1},
$$

and thus from 2.5 we get

$$
\begin{aligned}
\tilde{s}_{j} & =s_{j}-\mu_{2 j}+v_{j, 2 j-1}^{H} H_{j}^{-1} v_{j, 2 j-1}+\tilde{\mu}_{2 j}-\tilde{v}_{j, 2 j-1}^{H} \tilde{H}_{j, k}^{-1} \tilde{v}_{j, 2 j-1} \\
& =s_{j}-v_{j, 2 j-1}^{H}\left(\tilde{H}_{j, k}^{-1}-H_{j}^{-1}\right) \tilde{v}_{j, 2 j-1} .
\end{aligned}
$$

From 4.5 and using (2.6), we deduce 4.10). On the other hand, for $j \leq k \leq 2 j-1$ we have

$$
\tilde{\mu}_{2 j}=\mu_{2 j} \quad \text { and } \quad \tilde{v}_{j, 2 j-1}=v_{j, 2 j-1}+\chi_{k-j}^{(j)} \mathbf{M}_{k} .
$$

From (2.5) and (2.6), proceeding as above, we get

$$
\begin{aligned}
\tilde{s}_{j} & =s_{j}-\mu_{2 j}+v_{j, 2 j-1}^{H} H_{j}^{-1} v_{j, 2 j-1}+\tilde{\mu}_{2 j}-\tilde{v}_{j, 2 j-1}^{H} \tilde{H}_{j, k}^{-1} \tilde{v}_{j, 2 j-1} \\
& =s_{j}-v_{j, 2 j-1}^{H}\left(\tilde{H}_{j, k}^{-1}-H_{j}^{-1}\right) \tilde{v}_{j, 2 j-1}+\frac{\left(P_{j}^{(k-j)}(0)\right)^{H}}{(k-j) !} \mathbf{M}_{k}+\mathbf{M}_{k} \frac{\tilde{P}_{j}^{(k-j)}(0)}{(k-j) !},
\end{aligned}
$$

and from 4.6 and using 2.6,

$$
\begin{aligned}
\tilde{s}_{j}=s_{j}+\sum_{p=k-(j-1)}^{j-1} \frac{\left(P_{j}^{(k-p)}(0)\right)^{H}}{(k-p) !} \mathbf{M}_{k} \frac{\tilde{P}_{j}^{(p)}(0)}{p !} & +\frac{\left(P_{j}^{(k-j)}(0)\right)^{H}}{(k-j) !} \mathbf{M}_{k} \frac{\tilde{P}_{j}^{(j)}(0)}{j !} \\
& +\frac{\left(P_{j}^{(j)}(0)\right)^{H}}{j !} \mathbf{M}_{k} \frac{\tilde{P}_{j}^{(k-j)}(0)}{(k-j) !} \\
= & s_{j}+\sum_{p=k-j}^{j} \frac{\left(P_{j}^{(k-p)}(0)\right)^{H}}{(k-p) !} \mathbf{M}_{k} \frac{\tilde{P}_{j}^{(p)}(0)}{p !} .
\end{aligned}
$$

Since

$$
\begin{cases}\tilde{P}_{j}^{(p)}(0)=\mathbf{0}, & \text { if } j<p, \\ P_{j}^{(k-p)}(0)=\mathbf{0}, & \text { if } p<k-j\end{cases}
$$


we get 4.10 . Finally, for $k=2 j$ (notice that if $j=n$ the result is also valid with $\left.\mathbf{M}_{2 j}=\mathbf{0}\right)$,

$$
\tilde{\mu}_{2 j}=\mu_{2 j}+\mathbf{M}_{2 j} \quad \text { and } \quad \tilde{v}_{j, 2 j-1}^{H} \tilde{H}_{j, k}^{-1} \tilde{v}_{j, 2 j-1}=v_{j, 2 j-1}^{H} H_{j}^{-1} v_{j, 2 j-1},
$$

and thus from 2.5 ,

$$
\begin{aligned}
\tilde{s}_{j} & =s_{j}-\mu_{2 j}+v_{j, 2 j-1}^{H} H_{j}^{-1} v_{j, 2 j-1}+\tilde{\mu}_{2 j}-\tilde{v}_{j, 2 j-1}^{H} \tilde{H}_{j, k}^{-1} \tilde{v}_{j, 2 j-1} \\
& =s_{j}-\mu_{2 j}+\tilde{\mu}_{2 j}=s_{j}+\mathbf{M}_{2 j}=s_{j}+\sum_{p=0}^{k} \frac{\left(P_{j}^{(k-p)}(0)\right)^{H}}{(k-p) !} \mathbf{M}_{k} \frac{\tilde{P}_{j}^{(p)}(0)}{p !} .
\end{aligned}
$$

On the other hand, using the notation given in 4.1, 4.10 can be written as

$$
\tilde{s}_{j}=s_{j}+\left[P_{j}^{(k)}(0)^{H} P_{j}^{(k-1)}(0)^{H} \cdots P_{j}^{(0)}(0)^{H}\right] \mathbf{D}_{k, 0}^{-1} \mathbf{D}_{\mathbf{M}_{k}} \mathbf{D}_{0, k}^{-1} \tilde{\mathbf{P}}_{j, k}(0),
$$

which, using 4.2 and 4.3 , becomes

$$
\tilde{s}_{j}=s_{j}+\mathbf{P}_{j, k}(0)^{H} \Lambda_{k, 0}^{(k+1)} \mathbb{M}(k, j) \mathbf{P}_{j, k}(0) .
$$

The general case, i.e. when all moments are perturbed $(k=0, \ldots, 2 n-1)$ is easy to deduce, since we have 4.8 and

$$
\tilde{v}_{j, 2 j-1}=v_{j, 2 j-1}+\sum_{k=0}^{2 j-1} \chi_{k-j}^{(j)} \mathbf{M}_{k},
$$

so 4.9 follows.

\section{Connection Formulas For the Unit CIRCle AND Further RESUlts}

Let $\sigma$ be a Hermitian matrix measure supported on $\mathbb{T}$, with associated monic matrix orthogonal polynomials $\left(\Phi_{n}(z)\right)_{n \geq 0}$ and matrix moments $\left(c_{n}\right)_{n \geq 0}$. We define a new Hermitian sequence of matrix moments by $\left(\tilde{c}_{n}\right)_{n \geq 0}$, where $\tilde{c}_{n}=c_{n}+m_{n} \in \mathcal{M}_{l}$ for $n \geq 0$, and denote its corresponding Hermitian matrix measure and monic matrix orthogonal polynomials by $\tilde{\sigma}$ and $\left(\tilde{\Phi}_{n}(z)\right)_{n \geq 0}$, respectively. Furthermore, we define

$$
S_{j}=\left\langle\Phi_{j}, \Phi_{j}\right\rangle_{R, \sigma} \quad \text { and } \quad \tilde{S}_{j}=\left\langle\tilde{\Phi}_{j}, \tilde{\Phi}_{j}\right\rangle_{R, \tilde{\sigma}}
$$

In this section, in a similar way as in the real case, we will show the relation between the matrix polynomials $\tilde{\Phi}_{j}$ and $\Phi_{j}$, as well as the relation between $\tilde{S}_{j}$ and $S_{j}$, in terms of the matrix moments $\left(c_{n}\right)_{n \geq 0}$. Notice that 3.5 constitutes a particular case, when only one of the moments is perturbed. Let us define the following notation:

$$
\begin{aligned}
\mathbb{K}_{j-1}^{k}(0, z) & :=\left[K_{j-1}^{(0,0)}(0, z) K_{j-1}^{(1,0)}(0, z) \cdots K_{j-1}^{(k, 0)}(0, z)\right] \in \mathcal{M}_{l \times(k+1) l}, \\
\Upsilon_{j}^{k}(0) & :=\left[\Phi_{j}^{(0)}(0)^{T} \Phi_{j}^{(1)}(0)^{T} \cdots \Phi_{j}^{(k)}(0)^{T}\right]^{T} \in \mathcal{M}_{(k+1) l \times l}, \\
\tilde{\Upsilon}_{j}^{k}(0) & :=\left[\tilde{\Phi}_{j}^{(0)}(0)^{T} \tilde{\Phi}_{j}^{(1)}(0)^{T} \cdots \tilde{\Phi}_{j}^{(k)}(0)^{T}\right]^{T} \in \mathcal{M}_{(k+1) l \times l}, \\
\mathbf{D}_{m_{k}}^{j+1} & :=\operatorname{diag}\left\{m_{k} \cdots m_{k}\right\} \in \mathcal{M}_{(j+1) l}, \\
\mathbf{D}_{k, j+k} & :=\operatorname{diag}\{k ! \mathbf{I} \cdots(j+k) ! \mathbf{I}\} \in \mathcal{M}_{(j+1) l} .
\end{aligned}
$$


As in the real line case, for a fixed $k$, with $0 \leq k \leq j-1$, it has been proved in 6 ] that

$$
\begin{aligned}
\tilde{\Phi}_{j}(z)= & \Phi_{j}(z)-\sum_{p=0}^{j-k} \frac{K_{j-1}^{(p, 0)}(0, z)}{p !} m_{k} \frac{\tilde{\Phi}_{j}^{(p+k)}(0)}{(p+k) !}-\sum_{p=0}^{j-k-1} \frac{K_{j-1}^{(p+k, 0)}(0, z)}{(p+k) !} m_{k}^{H} \frac{\tilde{\Phi}_{j}^{(p)}(0)}{p !} \\
= & \Phi_{j}(z) \\
& -\mathbb{K}_{j-1}^{j}(0, z)\left(\mathbf{D}_{0, j}^{-1} \mathbf{D}_{k, j+k}^{-1} \Lambda_{-k, j-k}^{(j+1)} \mathbf{D}_{m_{k}}^{j+1}+\Lambda_{k, j+k}^{(j+1)} \mathbf{D}_{0, j}^{-1} \mathbf{D}_{k, j+k}^{-1} \mathbf{D}_{m_{k}^{H}}^{j+1}\right) \tilde{\Upsilon}_{j}^{j}(0),
\end{aligned}
$$

with

$$
\begin{aligned}
& \tilde{\Upsilon}_{j}^{j}(0)= \\
& \begin{aligned}
\left(\mathbf{I}_{(j+1) l}+\mathbf{D}_{0, j}\left(\Lambda_{0, j}^{(j)}\right)^{H} T_{j}^{-1} \Lambda_{0, j}^{(j)}\left(\mathbf{D}_{k, j+k}^{-1} \Lambda_{-k, j-k}^{(j+1)} \mathbf{D}_{m_{k}}^{j+1}+\Lambda_{k, j+k}^{(j+1)} \mathbf{D}_{0, j}^{-1} \mathbf{D}_{m_{k}^{H}}^{j+1}\right)\right)^{-1} \\
\times \Upsilon_{j}^{j}(0) .
\end{aligned}
\end{aligned}
$$

As a consequence, we have the following result.

Theorem 5.1 ([6]). Let $\sigma$ be a Hermitian matrix measure supported on $\mathbb{T}$ with matrix moments $\left(c_{k}\right)_{k=0}^{n}$. Define a new Hermitian sequence of matrix moments $\left(\tilde{c}_{k}\right)_{k=0}^{n}$ by $\tilde{c}_{k}=c_{k}+m_{k}$, for $0 \leq k \leq n$. Then, if $\left(\Phi_{j}(z)\right)_{j=0}^{n}$ and $\left(\tilde{\Phi}_{j}(z)\right)_{j=0}^{n}$ are the corresponding monic matrix orthogonal polynomials, we have

$$
\tilde{\Phi}_{j}(z)=\Phi_{j}(z)-\sum_{k=0}^{j} \mathbb{K}_{j-1}^{j}(0, z) \mathbb{N}(k, j) \Upsilon_{j}^{j}(0), \quad \text { with } \tilde{\Phi}_{0}(x)=\Phi_{0}(x)=\boldsymbol{I}
$$

where

$$
\begin{aligned}
& \mathbb{N}(k, j)=\left(\boldsymbol{D}_{0, j}^{-1} \boldsymbol{D}_{k, j+k}^{-1} \Lambda_{-k, j-k}^{(j+1)} \boldsymbol{D}_{m_{k}}^{j+1}+\Lambda_{k, j+k}^{(j+1)} \boldsymbol{D}_{0, j}^{-1} \boldsymbol{D}_{k, j+k}^{-1} \boldsymbol{D}_{m_{k}^{H}}^{j+1}\right) \\
& \times\left(\boldsymbol{I}_{(j+1) l}+\boldsymbol{D}_{0, j}\left(\Lambda_{0, j}^{(j)}\right)^{H} T_{j}^{-1} \Lambda_{0, j}^{(j)}\left(\boldsymbol{D}_{k, j+k}^{-1} \Lambda_{-k, j-k}^{(j+1)} \boldsymbol{D}_{m_{k}}^{j+1}+\Lambda_{k, j+k}^{(j+1)} \boldsymbol{D}_{0, j}^{-1} \boldsymbol{D}_{m_{k}^{H}}^{j+1}\right)\right)^{-1}
\end{aligned}
$$

The above theorem is a generalization to the matrix case of what was studied in [4, 19. The particular case of a perturbation of a single matrix moment given by (3.5) is shown in the following corollary.

Corollary 5.2. Let $k \in \mathbb{N}$ (fixed) with $0 \leq k \leq n$, and let $\sigma$ be a Hermitian matrix measure supported on $\mathbb{T}$. Define $\sigma_{k}$ as a perturbation of $\sigma$ given by (3.5). Then,

$$
\tilde{\Phi}_{j}(z)= \begin{cases}\Phi_{j}(z), & 0 \leq j<k, \\ \Phi_{j}(z)-\mathbb{K}_{j-1}^{j}(0, z) \mathbb{N}(k, j) \Upsilon_{j}^{j}(0), & k \leq j \leq n,\end{cases}
$$

with $\mathbb{N}(k, j)$ as in $(5.3)$. 
On the other hand, for $1 \leq j \leq n$, let $\tilde{\mathbf{N}}_{j} \in \mathcal{M}_{j l}$ be defined as

$$
\tilde{\mathbf{N}}_{j}:=\left[\begin{array}{ccccc}
m_{0}^{H}+m_{0} & \cdots & m_{k} & \cdots & m_{j-1} \\
\vdots & \ddots & & \ddots & \\
m_{k}^{H} & & m_{0}^{H}+m_{0} & & m_{k} \\
\vdots & \ddots & & \ddots & \\
m_{j-1}^{H} & \cdots & m_{k}^{H} & \cdots & m_{0}^{H}+m_{0}
\end{array}\right]=\mathbf{N}_{j, 0}+\cdots+\mathbf{N}_{j, j-1},
$$

where for $k=0, \ldots, j-1$, we have

$$
\mathbf{N}_{j, k}:=\left[\begin{array}{ccccc}
\mathbf{0} & \cdots & m_{k} & \cdots & \mathbf{0} \\
\vdots & \ddots & & \ddots & \vdots \\
\mathbf{0} & & \vdots & & m_{k} \\
\vdots & & & \ddots & \\
\mathbf{0} & \cdots & & \cdots & \mathbf{0}
\end{array}\right]+\left[\begin{array}{ccccc}
\mathbf{0} & \cdots & \mathbf{0} & \cdots & \mathbf{0} \\
\vdots & \ddots & & & \vdots \\
m_{k}^{H} & & \vdots & & \vdots \\
\vdots & \ddots & & \ddots & \\
\mathbf{0} & \cdots & m_{k}^{H} & \cdots & \mathbf{0}
\end{array}\right] \text {, }
$$

and for $k \geq j, \mathbf{N}_{j, k}=\mathbf{0} \in \mathcal{M}_{l j}$.

Lemma 5.3. For $1 \leq j \leq n$ and $0 \leq k \leq j-1$, define

$$
\tilde{T}_{j, k}=T_{j}+\boldsymbol{N}_{j, k} .
$$

Then,

$$
T_{j}^{-1}-\tilde{T}_{j, k}^{-1}=\sum_{p=0}^{j-k-1} T_{j}^{-1} \chi_{p}^{(j)} m_{k}\left(\chi_{p+k}^{(j)}\right)^{H} \tilde{T}_{j, k}^{-1}+\sum_{p=0}^{j-k-1} T_{j}^{-1} \chi_{p+k}^{(j)} m_{k}^{H}\left(\chi_{p}^{(j)}\right)^{H} \tilde{T}_{j, k}^{-1} .
$$

Proof. We have

$$
\tilde{T}_{j, k}^{-1}-T_{j}^{-1}=T_{j}^{-1}\left(T_{j}-\tilde{T}_{j, k}\right) \tilde{T}_{j, k}^{-1}=-T_{j}^{-1} \mathbf{N}_{j, k} \tilde{T}_{j, k}^{-1} .
$$

Notice that

$$
\mathbf{N}_{j, k}=\sum_{p=0}^{j-k-1} \chi_{p}^{(j)} m_{k}\left(\chi_{p+k}^{(j)}\right)^{H}+\sum_{p=0}^{j-k-1} \chi_{p+k}^{(j)} m_{k}^{H}\left(\chi_{p}^{(j)}\right)^{H},
$$

and thus substituting $\mathbf{N}_{j, k}$ in 5.6, 5.5 follows.

Let $\tilde{T}_{j} \in \mathcal{M}_{l j}$ be the block Toeplitz matrix with every moment perturbed, i.e.,

$$
\tilde{T}_{j}=\left[\begin{array}{cccc}
\tilde{c}_{0}^{H}+\tilde{c}_{0} & \tilde{c}_{1} & \cdots & \tilde{c}_{j-1} \\
\tilde{c}_{1}^{H} & \tilde{c}_{0}^{H}+\tilde{c}_{0} & \cdots & \tilde{c}_{j-2} \\
\vdots & \vdots & \ddots & \vdots \\
\tilde{c}_{j-1}^{H} & \tilde{c}_{j-2}^{H} & \cdots & \tilde{c}_{0}^{H}+\tilde{c}_{0}
\end{array}\right]=T_{j}+\tilde{\mathbf{N}}_{j} .
$$

Then, from (5.4), for $1 \leq j \leq n$, we get

$$
\tilde{T}_{j}=\tilde{T}_{j, 0}+\cdots+\tilde{T}_{j, j-1}
$$


Theorem 5.4. Let $\sigma$ be a Hermitian matrix measure supported on $\mathbb{T}$ with matrix moments $\left(c_{k}\right)_{k=0}^{n}$. Define a Hermitian sequence $\left(\tilde{c}_{k}\right)_{k=0}^{n}$ with $\tilde{c}_{k}=c_{k}+m_{k}$, for $0 \leq k \leq n$, and denote by $\tilde{\sigma}$ the associated Hermitian matrix measure. Then, for $0 \leq j \leq n$, we have

$$
\tilde{S}_{j}=S_{j}+\sum_{k=0}^{j} \Upsilon_{j}^{j}(0)^{H} \mathbb{N}(k, j) \Upsilon_{j}^{j}(0)
$$

with $\tilde{S}_{0}=\tilde{c}_{0}^{H}+\tilde{c}_{0}=c_{0}+m_{0}+\left(c_{0}+m_{0}\right)^{H}$ and $\mathbb{N}(k, j)$ as in 5.3.

Proof. Let us first assume that only a single $k$-th matrix moment is perturbed. From 2.11, we have that if $j<k$, then $\tilde{S}_{j}=S_{j}$. Let us show that for $0 \leq k \leq j$,

$$
\tilde{S}_{j}=S_{j}+\sum_{p=0}^{j-k} \frac{\left(\Phi_{j}^{(p)}(0)\right)^{H}}{p !} m_{k} \frac{\tilde{\Phi}_{j}^{(p+k)}(0)}{(p+k) !}+\sum_{p=0}^{j-k} \frac{\left(\Phi_{j}^{(p+k)}(0)\right)^{H}}{(p+k) !} m_{k}^{H} \frac{\tilde{\Phi}_{j}^{(p)}(0)}{p !} .
$$

For $k=0, \tilde{\nu}_{j}=\nu_{j}$, and from 2.11 we get

$$
\begin{aligned}
\tilde{S}_{j} & =\tilde{c}_{0}^{H}+\tilde{c}_{0}-\tilde{\nu}_{j}^{H} \tilde{T}_{j, k}^{-1} \tilde{\nu}_{j}=S_{j}-c_{0}^{H}-c_{0}+\nu_{j}^{H} T_{j}^{-1} \nu_{j}+\tilde{c}_{0}^{H}+\tilde{c}_{0}-\tilde{\nu}_{j}^{H} \tilde{T}_{j, k}^{-1} \tilde{\nu}_{j} \\
& =S_{j}+m_{0}^{H}+m_{0}-\nu_{j}\left(\tilde{T}_{j, k}^{-1}-T_{j}^{-1}\right) \tilde{\nu}_{j} .
\end{aligned}
$$

By using 2.12 and (5.5), we obtain

$$
\begin{aligned}
\tilde{S}_{j}= & S_{j}+m_{0}^{H}+m_{0} \\
& +\sum_{p=0}^{j-k-1} \frac{\left(\Phi_{j}^{(p)}(0)\right)^{H}}{p !} m_{k} \frac{\tilde{\Phi}_{j}^{(p+k)}(0)}{(p+k) !}+\sum_{p=0}^{j-k-1} \frac{\left(\Phi_{j}^{(p+k)}(0)\right)^{H}}{(p+k) !} m_{k}^{H} \frac{\tilde{\Phi}_{j}^{(p)}(0)}{p !},
\end{aligned}
$$

and since for $k=0$ we have

$$
m_{0}=\frac{\left(\Phi_{j}^{(j)}(0)\right)^{H}}{j !} m_{k} \frac{\tilde{\Phi}_{j-k+k}^{(j)}(0)}{(j-k+k) !}, \quad m_{0}^{H}=\frac{\left(\Phi_{j}^{(j-k+k)}(0)\right)^{H}}{(j-k+k) !} m_{k}^{H} \frac{\tilde{\Phi}_{j}^{(j)}(0)}{j !},
$$

we deduce 5.9 ).

For $1 \leq k \leq j, \tilde{\nu}_{j}=\nu_{j}+\chi_{j-k}^{(j)} m_{k}$, and proceeding as above we get

$$
\tilde{S}_{j}=S_{j}-\nu_{j}^{H}\left(\tilde{T}_{j, k}^{-1}-T_{j}^{-1}\right) \tilde{\nu}_{j}-\nu_{j}^{H} T_{j}^{-1} \chi_{j-k}^{(j)} m_{k}-m_{k}^{H}\left(\chi_{j-k}^{(j)}\right)^{H} \tilde{T}_{j, k}^{-1} \tilde{\nu}_{j} .
$$

By using 2.12 and (5.5), we obtain

$$
\begin{aligned}
\tilde{S}_{j}=S_{j} & +\sum_{p=0}^{j-k-1} \frac{\left(\Phi_{j}^{(p)}(0)\right)^{H}}{p !} m_{k} \frac{\tilde{\Phi}_{j}^{(p+k)}(0)}{(p+k) !}+\frac{\left(\Phi_{j}^{(j-k)}(0)\right)^{H}}{(j-k) !} m_{k} \frac{\tilde{\Phi}_{j}^{(j)}(0)}{j !} \\
& +\sum_{p=0}^{j-k-1} \frac{\left(\Phi_{j}^{(p+k)}(0)\right)^{H}}{(p+k) !} m_{k}^{H} \frac{\tilde{\Phi}_{j}^{(p)}(0)}{p !}+\frac{\left(\Phi_{j}^{(j)}(0)\right)^{H}}{j !} m_{k}^{H} \frac{\tilde{\Phi}_{j}^{(j-k)}(0)}{(j-k) !}
\end{aligned}
$$

so 5.9 follows. 
On the other hand, using (5.1), the first sum of $(5.9)$ can be written as

$$
\begin{aligned}
\sum_{p=0}^{j-k} \frac{\left(\Phi_{j}^{(p)}(0)\right)^{H}}{p !} m_{k} \frac{\tilde{\Phi}_{j}^{(p+k)}(0)}{(p+k) !} & =\sum_{p=k}^{j} \frac{\left(\Phi_{j}^{(p-k)}(0)\right)^{H}}{(p-k) !} m_{k} \frac{\tilde{\Phi}_{j}^{(p)}(0)}{p !} \\
& =\sum_{p=0}^{j} \frac{\left(\Phi_{j}^{(p-k)}(0)\right)^{H}}{(p-k) !} m_{k} \frac{\tilde{\Phi}_{j}^{(p)}(0)}{p !} \\
& =\Upsilon_{j}^{j}(0)^{H} \mathbf{D}_{0, j}^{-1} \mathbf{D}_{k, j+k}^{-1} \Lambda_{-k, j-k}^{(j+1)} \mathbf{D}_{m_{k}}^{j+1} \tilde{\Upsilon}_{j}^{j}(0)
\end{aligned}
$$

and in a similar way, the second sum of $(5.9)$ becomes

$$
\sum_{p=0}^{j-k} \frac{\left(\Phi_{j}^{(p+k)}(0)\right)^{H}}{(p+k) !} m_{k}^{H} \frac{\tilde{\Phi}_{j}^{(p)}(0)}{p !}=\Upsilon_{j}^{j}(0)^{H} \Lambda_{k, j+k}^{(j+1)} \mathbf{D}_{0, j}^{-1} \mathbf{D}_{k, j+k}^{-1} \mathbf{D}_{m_{k}^{H}}^{j+1} \tilde{\Upsilon}_{j}^{j}(0)
$$

As a consequence, 5.9 becomes

$$
\tilde{S}_{j}=S_{j}+\Upsilon_{j}^{j}(0)^{H}\left(\mathbf{D}_{0, j}^{-1} \mathbf{D}_{k, j+k}^{-1} \Lambda_{-k, j-k}^{(j+1)} \mathbf{D}_{m_{k}}^{j+1}+\Lambda_{k, j+k}^{(j+1)} \mathbf{D}_{0, j}^{-1} \mathbf{D}_{k, j+k}^{-1} \mathbf{D}_{m_{k}^{H}}^{j+1}\right) \tilde{\Upsilon}_{j}^{j}(0)
$$

which, using (5.2) and (5.3), can be written as

$$
\tilde{S}_{j}=S_{j}+\Upsilon_{j}^{j}(0)^{H} \mathbb{N}(k, j) \Upsilon_{j}^{j}(0)
$$

The general case, when all moments are perturbed, follows immediately taking into account (5.7) and

$$
\tilde{\nu}_{j}=\nu_{j}+\sum_{k=1}^{j} \chi_{j-k}^{(j)} m_{k}
$$

so 5.8 follows.

For the particular case of 3.5, we have

Corollary 5.5. Let $k \in \mathbb{N}$ (fixed) with $0 \leq k \leq n$, and let $\sigma$ be a Hermitian matrix measure supported on $\mathbb{T}$. Let $\sigma_{k}$ be a perturbation of $\sigma$ defined by (3.5). Then, for $0 \leq j \leq n$,

$$
\tilde{S}_{j}= \begin{cases}S_{j}, & 0 \leq j<k \\ S_{j}+\Upsilon_{j}^{j}(0)^{H} \mathbb{N}(k, j) \Upsilon_{j}^{j}(0), & k \leq j \leq n\end{cases}
$$

with $\mathbb{N}(k, j)$ as in 5.3 .

\section{Example}

To illustrate the results of the above subsections, consider the matrix measure that appears in [3, Section 8]:

$$
d \sigma(\theta)=\frac{1}{\sqrt{\cos 2 \theta}}\left[\begin{array}{cc}
\sqrt{1+\cos 2 \theta} & \pm 1 \\
\pm 1 & \sqrt{1+\cos 2 \theta}
\end{array}\right] \frac{d \theta}{2 \pi}, \quad \text { if }\left\{\begin{array}{l}
\theta \in\left[-\frac{\pi}{4}, \frac{\pi}{4}\right] \\
\theta \in\left[\frac{3 \pi}{4}, \frac{5 \pi}{4}\right]
\end{array}\right.
$$


Its corresponding matrix moments are given by

$$
c_{0}=\mathbf{I}_{2}, c_{4 m}=c_{4 m+2}=\frac{\alpha_{m}}{2} \mathbf{I}_{2}, c_{4 m+1}=\frac{\alpha_{m}}{\sqrt{2}}\left[\begin{array}{ll}
0 & 1 \\
1 & 0
\end{array}\right], c_{4 m+3}=\mathbf{0}_{2},
$$

with

$$
\alpha_{0}=1, \quad \alpha_{n}=(-1)^{n} \prod_{k=1}^{n}\left(1-\frac{1}{2 k}\right), n \geq 1 .
$$

Now, we consider the problem of determining the obtained matrix measure if some of the moments given by 6.2 change. For instance, let us introduce a perturbation of the matrix moment $c_{4 m+1}$, for some $m \in \mathbb{Z}$, given by

$$
\tilde{c}_{4 m+1}=\frac{\tilde{\alpha}_{m}}{\sqrt{2}}\left[\begin{array}{ll}
0 & 1 \\
1 & 0
\end{array}\right]=\frac{\alpha_{m}+\epsilon}{\sqrt{2}}\left[\begin{array}{ll}
0 & 1 \\
1 & 0
\end{array}\right]=c_{4 m+1}+\frac{\epsilon}{\sqrt{2}}\left[\begin{array}{ll}
0 & 1 \\
1 & 0
\end{array}\right],
$$

i.e., $m_{4 m+1}=\frac{\epsilon}{\sqrt{2}}\left[\begin{array}{ll}0 & 1 \\ 1 & 0\end{array}\right]$. The perturbed matrix measure, obtained by using (3.6) for $j=4 m+1$, is

$$
d \sigma_{4 m+1}(\theta)=d \sigma(\theta)+\sqrt{2} \epsilon\left[\begin{array}{cc}
0 & \cos (4 m+1) \theta \\
\cos (4 m+1) \theta & 0
\end{array}\right] \frac{d \theta}{2 \pi}
$$

where $\sigma$ is as in 6.1. Notice that $\sqrt{2} \epsilon\left[\begin{array}{cc}0 & \cos (4 m+1) \theta \\ \cos (4 m+1) \theta & 0\end{array}\right]$ is a positive semi-definite matrix for $\theta \in(-\pi, \pi]$ if $\epsilon<0$. In particular, it is positive semi-definite in the same support as $\sigma$ and thus $\sigma_{4 m+1}$ is positive semi-definite if $\epsilon<0$. Furthermore, the matrix measure (6.1) and its perturbation 6.3 are Hermitian and thus there exists an associated sequence of orthogonal matrix polynomials, as well as their norms, given by Theorems 5.1 and 5.4

On the other hand, to illustrate the results in Section 3, by using (3.2), (3.3) and taking into account that $\cos 2 \theta=2 \cos ^{2} \theta-1$, we get

$$
d \mu(x)=S z^{-1}(d \sigma(\theta))=\frac{1}{\pi} \frac{1}{\sqrt{2 x^{2}-1}}\left[\begin{array}{cc}
\sqrt{2}|x| & \pm 1 \\
\pm 1 & \sqrt{2}|x|
\end{array}\right] \frac{d x}{\sqrt{1-x^{2}}},
$$

where the plus sign +1 holds in the interval $\left[\frac{1}{\sqrt{2}}, 1\right]$ and the minus sign -1 holds in the interval $\left[-1,-\frac{1}{\sqrt{2}}\right]$. 6.4 is the measure associated with the measure 6.1, connected through the inverse Szegö matrix transformation. Furthermore, from Proposition 3.1 with $\epsilon<0$ we get

$$
d \mu_{4 m+1}(x)=d \mu(x)+\frac{\sqrt{2} \epsilon}{\pi}\left[\begin{array}{cc}
0 & T_{4 m+1}(x) \\
T_{4 m+1}(x) & 0
\end{array}\right] \frac{d x}{\sqrt{1-x^{2}}},
$$

where $\mu$ is as in 6.4. Notice that the matrix measure (6.4) and its perturbation 6.5) are positive semi-definite, so there exists a corresponding sequence of matrix orthogonal polynomials on the real line and their norms, that can be obtained from Theorems 4.1 and 4.3 . 


\section{ACKNOWLEDGMENTS}

The authors would like to thank the anonymous referee for his/her very useful comments that helped to improve the paper.

\section{REFERENCES}

[1] Berezanskĭ Ju. M., Expansions in eigenfuntions of selfadjoint operators, Translated from the Russian by R. Bolstein, J. M. Danskin, J. Rovnyak and L. Shulman. Translations of Mathematical Monographs, Vol. 17, American Mathematical Society, Providence, R.I., 1968. MR0222718

[2] Bultheel A., Cantero M. J. and Cruz-Barroso R., Matrix methods for quadrature formulas on the unit circle. A survey. J. Comput. Appl. Math. 284 (2015), 78-100. MR3319496

[3] Cantero M. J., Grünbaum F. A., Moral L. and Velázquez L., Matrix-valued Szegő polynomials and quantum random walks, Comm. Pure Appl. Math. 63 (2010), no. 4, 464-507. MR2604869

[4] Castillo K., Garza L. and Marcellán F., Perturbations on the subdiagonals of Toeplitz matrices, Linear Algebra Appl. 434 (2011), no. 6, 1563-1579. MR2775766

[5] Chihara T. S., An introduction to orthogonal polynomials, Gordon and Breach, New YorkLondon-Paris, 1978. MR0481884.

[6] Choque-Rivero A. E. and Garza L. E., Matrix orthogonal polynomials associated with perturbations of block Toeplitz matrices, Russ. Math. (Iz. VUZ) 61 (2017), no. 12, 57-69. MR 3889199

[7] Choque-Rivero A. E. and Garza L. E., Moment perturbation of matrix polynomials, Integral Transforms Spec. Funct. 26 (2015), no. 3, 177-191. MR3293037

[8] Damanik D., Pushnitski A. and Simon B., The analytic theory of matrix orthogonal polynomials, Surv. Approx. Theory 4 (2008), 1-85. MR2379691

[9] Dette H. and Wagener J., Matrix measures on the unit circle, moment spaces, orthogonal polynomials and the Geronimus relations, Linear Algebra Appl. 432 (2010), no. 7, 1609-1626. MR2592906

[10] Dette H. and Tomecki D., Determinants of block Hankel matrices for random matrix-valued measures, Stochastic Process. Appl. 129 (2019), no. 12, 5200-5235. MR 4025703

[11] Durán A. J., On orthogonal polynomials with respect to positive definite matrix of measures, Canad. J. Math. 47 (1995), no. 1, 88-112. MR1319691.

[12] Durán A. J. and Defez E., Orthogonal matrix polynomials and quadrature formulas, Linear Algebra Appl. 345 (2002), 71-84. MR1883268

[13] Durán A. J. and Grünbaum F. A., A survey on orthogonal matrix polynomials satisfying second order differential equations, J. Comput. Appl. Math. 178 (2005), no. 1-2, 169-190. MR2127878

[14] Durán A. J. and López-Rodríguez P., Density questions for the truncated matrix moment problem, Canad. J. Math. 49 (1997), no. 4, 708-721. MR1471052

[15] Durán A. J. and López-Rodríguez P., The $\mathcal{L}^{p}$ space of a positive definite matrix of measures and density of matrix polynomials in $\mathcal{L}^{1}$, J. Approx. Theory 90 (1997), no. 2, 299-318. MR1462266

[16] Durán A. J. and Polo B., Gaussian quadrature formulae for matrix weights, Linear Algebra Appl. 355 (2002), 119-146. MR1930141

[17] Durán A. J. and Van Assche W., Orthogonal matrix polynomials and higher-order recurrence relations, Linear Algebra Appl. 219 (1995), 261-280. MR1327404

[18] Fuentes E. and Garza L. E., Analysis of perturbations of moments associated with orthogonality linear functionals through the Szegö transformation, Rev. Integr. Temas Mat. 33 (2015), no. 1, 61-82. MR3364658

[19] Fuentes E. and Garza L. E., On a finite moment perturbation of linear functionals and the inverse Szegö transformation, Rev. Integr. Temas Mat. 34 (2016), no. 1, 39-58. MR3524147. 
[20] Fuhrmann P. A., Orthogonal matrix polynomials and system theory, Rend. Sem. Mat. Univ. Politec. Torino, 1987, Special Issue, 68-124. MR0948972

[21] Krĕ̌n M., Infinite $J$-matrices and a matrix-moment problem (in Russian), Doklady Akad. Nauk SSSR (N.S.) 69 (1949), 125-128. MR0034964

[22] Krein M., On a generalization of some investigations of G. Szegő, V. Smirnoff, and A. Kolmogoroff, C. R. (Doklady) Acad. Sci. URSS (N.S.) 46 (1945), 91-94. MR0013457

[23] Kreyszig E., Introductory functional analysis with applications, John Wiley \& Sons, New York, 1989. MR0992618.

[24] López-Rodríguez P., Riesz's theorem for orthogonal matrix polynomials, Constr. Approx. 15 (1999), no. 1, 135-151. MR1660065

[25] López-Rodríguez P., The Nevanlinna parametrization for a matrix moment problem, Math. Scand. 89 (2001), no. 2, 245-267. MR1868176.

[26] Miranian L., Matrix valued orthogonal polynomials on the unit circle: some extensions of the classical theory, Canad. Math. Bull. 52 (2009), no. 1, 95-104. MR2494315.

[27] Miranian L., Matrix-valued orthogonal polynomials on the real line: some extensions of the classical theory, J. Phys. A 38 (2005), no. 25, 5731-5749. MR2168386.

[28] Morse P. M. and Feshbach H., Methods of theoretical physics, McGraw-Hill, New YorkToronto-London, 1953. MR0059774.

[29] Nikishin E. M., The discrete Sturm-Liouville operator and some problems of function theory (Russian) Trudy Sem. Petrovsk. 10 (1984), 3-77, 237. MR0778879

[30] Simon B., Orthogonal polynomials on the unit circle, Part 2. Spectral theory. American Mathematical Society Colloquium Publications, 54. American Mathematical Society, Providence, RI, 2005. MR2105089

[31] Sinap A., Gaussian quadrature for matrix valued functions on the unit circle, Electron. Trans. Numer. Anal. 3 (1995), 96-115. MR1353294.

[32] Stieltjes T.-J., Recherches sur les fractions continues, Ann. Fac. Sci. Toulouse Sci. Math. Sci. Phys. 8 (1894), no. 4, J1-J122. MR1508159

[33] Yakhlef H. O. and Marcellán F., Orthogonal matrix polynomials, connection between recurrences on the unit circle and on a finite interval, in Approximation, optimization and mathematical economics (Pointe-à-Pitre, 1999), 369-382, Physica, Heidelberg, 2001. MR1842902.

\author{
E. Fuentes \\ Departamento de Matemáticas, Universidad Nacional de Colombia, Ciudad Universitaria, \\ Bogotá, Colombia \\ efuentes@unal.edu.co \\ L. E. Garza ${ }^{凶}$ \\ Facultad de Ciencias, Universidad de Colima, Bernal Díaz del Castillo 340, Colima, México \\ luis_garza1@ucol.mx
}

Received: October 5, 2018

Accepted: April 18, 2019 